\title{
GEOSTATISTICS FOR AIR QUALITY MAPPING: CASE OF BAGUIO CITY, PHILIPPINES
}

\author{
R. V. Ramos ${ }^{1}$, A.C. Blanco ${ }^{1}$ \\ ${ }^{1}$ Department of Geodetic Engineering, University of the Philippines, Diliman \\ Quezon City 1101 Philippines - (rvramos, acblanco) @up.edu.ph
}

\section{Commission IV}

KEY WORDS: Atmospheric Dispersion Model, GIS, Particulate Matter, Ordinary Kriging, ME, RMSE

\begin{abstract}
:
Mapping of air quality are often based on ground measurements using gravimetric and air portable sensors, remote sensing methods and atmospheric dispersion models. In this study, Geographic Information Systems (GIS) and geostatistical techniques are employed to evaluate coarse particulate matter $\left(\mathrm{PM}_{10}\right)$ concentrations observed in the Central Business District of Baguio City, Philippines. Baguio City has been reported as one of the most polluted cities in the country and several studies have already been conducted in monitoring its air quality. The datasets utilized in this study are based on hourly simulations from a Gaussian-based atmospheric dispersion model that considers the impacts of vehicular emissions. Dispersion modeling results, i.e., $\mathrm{PM}_{10}$ concentrations at 20 meter interval, show that high values range from 135 to $422 \mu \mathrm{g} / \mathrm{m}^{3}$. The pollutant concentrations are evident within 40 meters from the roads. Spatial variations and $\mathrm{PM}_{10}$ estimates at unsampled locations are determined using Ordinary Kriging. Geostatistical modeling estimates are evaluated based on recommended values for mean error (ME), root mean square error (RMSE) and standardized errors. Optimal predictors for pollutant concentrations at 5-meter interval include 2 to 5 search neighbors and variable smoothing factor for night-time datasets while 2 to 10 search neighbors and smoothing factors 0.3 to 0.5 were used for daytime datasets. Results from several interpolation tests indicate small ME $(0.0003$ to $0.0008 \mu \mathrm{g} / \mathrm{m} 3)$ and average standardized errors (4.24 to $8.67 \mu \mathrm{g} / \mathrm{m} 3$ ). RMSE ranged from 2.95 to $5.43 \mu \mathrm{g} / \mathrm{m} 3$, which are approximately 2 to $3 \%$ of the maximum pollutant concentrations in the area. The methodology presented in this paper may be integrated with atmospheric dispersion models in refining estimates of pollutant concentrations, in generating surface representations, and in understanding the spatial variations of the outputs from the model simulations.
\end{abstract}

\section{INTRODUCTION}

\subsection{Background of the Study}

Air quality monitoring activities in the Philippines has been undertaken by several agencies to generate significant information in formulating air pollution management and control programs. The Environmental Management Bureau (EMB) under the Department of Environment and Natural Resources (DENR) with other institutions such as the Manila Observatory, Philippine Nuclear Research Institute (PNRI) and the University of the Philippines Institute of Environmental Science and Meteorology (UP IESM) have been actively engaging in projects related to air quality monitoring with extensive resources and established monitoring stations in the country, most of which are located in the Metro Manila area. There are numerous efforts in monitoring air quality in Metro Manila by these agencies but only a few have been planned and implemented in other urban cities. Other urban cities that were mentioned by DENR EMB as priority areas for air quality monitoring are Baguio City, Cebu City, and Davao City. According to several news articles since 2014 (Comanda, 2018) (Peña, 2018)(Guieb, 2015)(Locsin, 2014), citing reports from the World Health Organization (WHO), Baguio City is one of the most polluted cities in the country. The Central Business District (CBD) of Baguio City has been identified as one with the highest air pollution concentration among the urban cities, even higher than cities in Metro Manila (Guieb, 2015). Despite the increase of the air quality monitoring stations in the country operated by EMB, the number of stations located in the Cordillera Administrative Region (CAR), in which Baguio City is included, is still limited to one. The continuous air quality monitoring station within the said region is located at Burnham Park in Baguio City. The CAR airshed covers Baguio City and the municipalities of La Trinidad, Itogon, Sablan, Tublay and Tuba (BLISTT). The Philippine Clean Air Act of 1999 or Republic Act 8749 indicates the designated airsheds, defined as areas with common weather or meteorological conditions and sources of air pollution that affect the interchange and diffusion of pollution, to facilitate the monitoring of air quality (EMB, 2004).

The City Environment and Parks Management Office (CEPMO) of the Local Government Unit (LGU) of Baguio City is incharge of monitoring ambient and roadside air quality. Air quality measurements recorded by CEPMO, however, is limited only to particulate matter (PM) concentrations. The PM measurements recorded by the continuous air quality monitoring station located at Burnham Park is used to describe the general trends of ambient air quality in Baguio City. These records do not specify portions of the city that are heavily polluted which may have significant impacts on the health of pedestrians and commuters. The unit has identified that transportation and air quality control measures are necessary to conform to the air quality guideline values for PM. The LGUBaguio made interventions through the implementation of number coding for vehicles plying in the city, anti-smoke belching units and roadside inspection and testing monitoring team (RITMT). Given these initiatives by the LGU, it is better to provide detailed information on the location of pollution concentrations to determine if the traffic schemes are helpful in improving air quality conditions in the CBD area. The LGU may use these types of information to assist in their planning for better transport management. 


\subsection{Research Objectives}

This study aims to determine the locations and distributions of the PM concentrations within CBD-Baguio City. The spatial and temporal variations of these pollutant concentrations are to be characterized using Geographic Information Systems (GIS)based geostatistical modeling.

\subsection{Scope and Limitations}

In Baguio City, the possible sources of PM identified in the CBD are soil sources, soil-road dust resuspension, and vehicular emissions (Hagad, 2018). This study will focus on quantifying vehicular emissions in CBD-Baguio City (refere to Figure 1) and will highlight how these emission sources vary spatially through different temporal scenarios, i.e. daytime and nighttime hours. The traffic and meteorological datasets utilized in this study were obtained on 2014. The simulation period is limited to 24-hours, from 12:00 midnight to 11:00 PM of February 8, 2014.

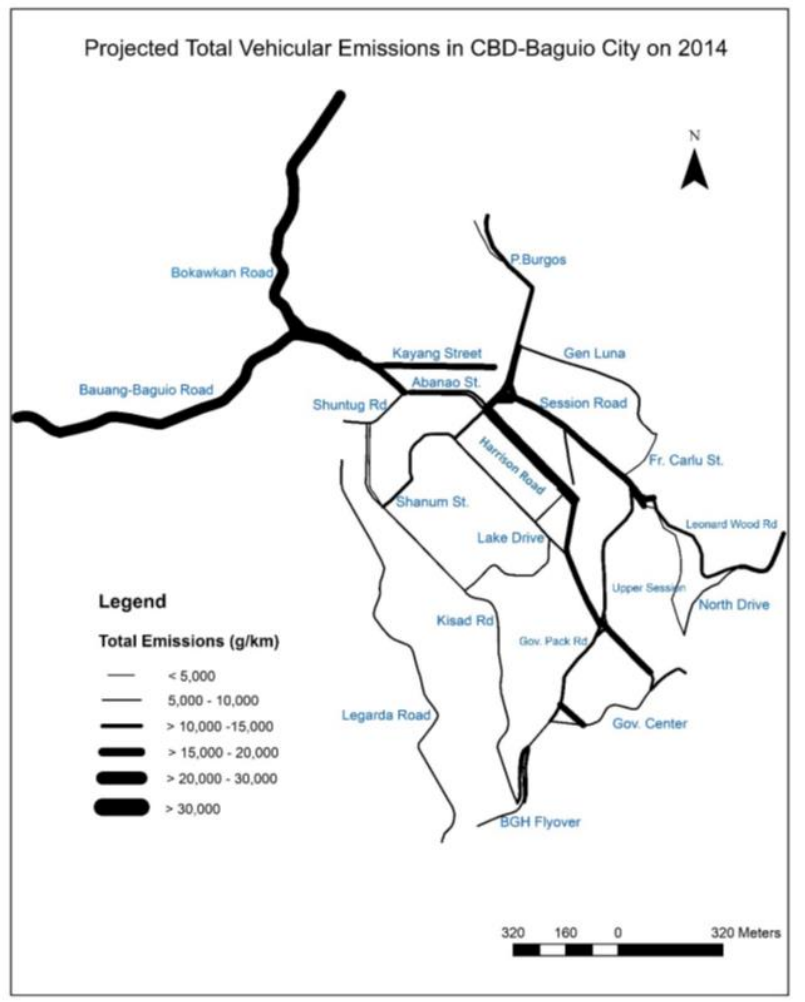

Figure 1. Total vehicular emissions on 2014 based on traffic projections and annual average daily traffic (AADT) in 2016

The PM measurements recorded at the Burnham Park station are for fine particulates $\left(\mathrm{PM}_{2.5}\right)$ and coarse particulates $\left(\mathrm{PM}_{10}\right)$. These particulates mainly differ on the size or diameter and their residence time in the atmosphere. $\mathrm{PM}_{10}$ tends to stay longer near the surface and contributes to build-up of pollutants in the ground. The dispersion models were designed for $\mathrm{PM}_{10}$ only, with the assumption that estimated concentrations resulting from vehicle exhaust emissions can be valid up to an altitude of 5 meters. In-situ measurements requested from UP IESM were obtained at 5-meter altitude. Parameterization of the dispersion model shall not be discussed in this paper. Validation of the emissions and dispersion modeling results is beyond the scope of this study. The accuracy of the geostatistical models is based on the assessment of prediction errors discussed in section 2.2.4.

\subsection{Review of Related Literature}

Transport and dispersion of air pollutants in urban areas vary depending on their source. Cities are generally the most affected areas with a critical concentration of airborne PM (Pospisil, Jicha, 2010). The concentration of PM in urban areas is the result of combinations of concentrations from different factors in the area such as regional background, urban and traffic (Shahraiyni, Sodoudi, 2016).

Monitoring of airborne PM and other air pollutants in urban areas is categorized into field measurements, laboratory experiments such as wind tunnel set-ups, empirical models and Computational Fluid Dynamics (CFD) techniques (Tominaga, Stathopoulos, 2016). Field measurements include data collection using gravimetric-based sensors, which are typically mobile and acquires samples in short time intervals, and wind tunnel experiments for large-scale and localized regions (Xia et al., 2014). Portable air quality sensors are used to collect samples of PM through filters that are harvested and weighed after a 24-hr sampling period. In-situ measurements may also be collected through fixed monitoring stations (Gulliver, Briggs, 2011) (Wu, Kuo, 2013) such as the regional monitoring stations maintained by the DENR-EMB in the Philippines.

Atmospheric dispersion models use mathematical equations that describe the dispersion, chemical, and physical processes within the plume to calculate the concentrations at various locations (Holmes, Morawska, 2006). Dispersion models for predicting PM concentrations in urban areas include deterministic and statistical models. Deterministic or mechanistic models involve numerically solving a set of differential equations to predict the spatio-temporal variations of a pollutant while statistical models are utilized in describing the complex site-specific relationship between air pollutants and explanatory variables (Shahraiyni, Sodoudi, 2016). These two approaches generally differ on the following: (1) data requirements, (2) information on the source of pollutant and (3) modeling period. Deterministic models often tend to have longer modeling period in terms of computational or processing time compared to statistical models. Since combinations of deterministic models can be utilized to estimate $\mathrm{PM}_{10}$ concentrations, it would take long hours of simulation than a single statistical model that can be developed easier and faster.

Deterministic models often possess an inaccurate description of the dispersion processes and may lead to significant bias and error in predicting pollutant concentrations variables (Shahraiyni, Sodoudi, 2016). With these limitations, GIS-based and geostatistical methods can improve the model estimates in terms of its spatial resolution and makes prediction less timeconsuming. Geostatistics is often used for wide areas with numerous air quality monitoring stations that can provide enough measurements for spatial and temporal analysis of pollutant concentrations. In the case of a sparse number of measurements, geostatistical techniques introduce its usefulness in up-scaling from the data that have been collected at limited points to provide complete areal coverage where the accuracy of the prediction is known (Sertel et al., 2006). Geostatistics provides an advanced methodology to quantify the spatial features of the target variables and enables spatial interpolation (Nas, 2009). Geostatistical models for uncertainty assessments have been developed and applied to environmental sciences particularly for characterizing polluted sites (Paper et al., 2000). Halimi et.al. (2016) investigated the spatial distribution of carbon monoxide $(\mathrm{CO})$, nitrogen dioxide and $\mathrm{PM}_{10}$ using different geostatistical interpolation methods applied on 
measurements from 21 air quality monitoring stations in Tehran, Iran. Moral García et al. (2010) carried out sampling campaigns in an urban area in Badajoz City, Spain to measure ambient ozone concentrations using an automatic portable analyzer. This study made use of geostatistical methods to analyze the distribution of ozone concentrations within the city. Enkhtur (2013) conducted modeling of $\mathrm{PM}_{10}$ concentrations within five northwestern European countries using geostatistical space-time approach integrating secondary information from different data sources. Prediction and probability maps were created in the same study based on Universal Kriging models.

Geostatistical methods using GIS are employed in this study to create detailed estimates of $\mathrm{PM}_{10}$ concentrations, which are generated from a $20 \times 20$ meter grid in the dispersion modeling process, through spatial interpolation. The main goal of spatial interpolation is to discern the spatial patterns of atmospheric pollution concentrations by estimating values at unsampled locations based on measurements at sample points (Moral García et al., 2010). Uncertainties of the estimates can also be assessed within the geostatistical modeling process.

Air pollution studies have employed distance-weighted techniques, but Kriging methods have been widely used in incorporating the spatial correlation into its estimation algorithm (Moral García et al., 2010). Kriging method is described as the best linear unbiased estimator and its estimates are based on the variogram model and measurements from sample points (Halimi et al., 2016). An advantage of the method is that it gives unbiased prediction with minimum variance and considers the spatial correlation between measurements at different locations (Moral García et al., 2010). The geostatistical techniques quantify the spatial autocorrelation among measured points and account for the spatial configuration of the sample points around the prediction location (Nas, 2009).

\section{METHODOLOGY}

\subsection{Datasets and Materials}

Geostatistical modeling is conducted to refine estimates of hourly $\mathrm{PM}_{10}$ concentrations resulting from Gaussian-based line source dispersion model simulations. The simulation results comprise of two (2) $20 \times 20$ gridded point locations of receptors with $\mathrm{PM}_{10}$ concentration values (refer to Figure 2). In order to check spatial variations of these pollutant concentrations within the roads, there is a need to calculate or estimate the $\mathrm{PM}_{10}$ concentrations at a smaller grid size. Mapping $\mathrm{PM}_{10}$ concentrations at a finer scale provide better representations of the values within and near the roads. The grid size for the geostatistical process is based on the smallest road width present in the model domain, which is 6 meters.

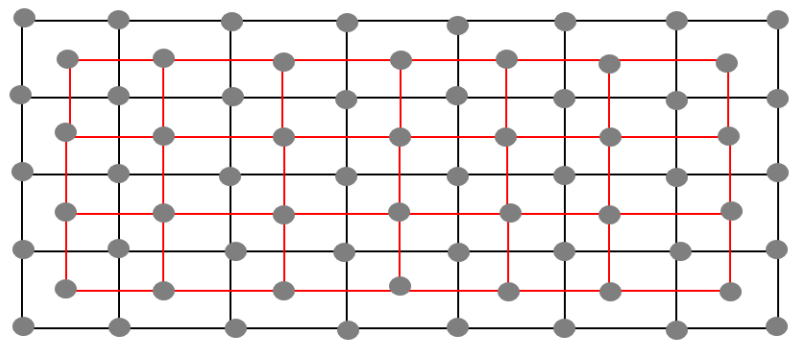

Figure 2. Receptor locations defined on corners (gray points) of two 20x20 meter grids (black and red grids)

\subsection{Geostatistical Modeling}

The geostatistical modeling process involves several steps in order to obtain detailed estimates of $\mathrm{PM}_{10}$ concentrations, particularly at $5 \times 5$ meter spatial resolution. The process is employed through the available Geostatistical Analyst toolbox in ArcGIS. The specific steps undertaken in this geostatistical modeling of $\mathrm{PM}_{10}$ concentrations are illustrated in Figure 3. Several interpolation tests are undertaken, and prediction errors are evaluated in each step to select the most appropriate parameters for the geostatistical model.

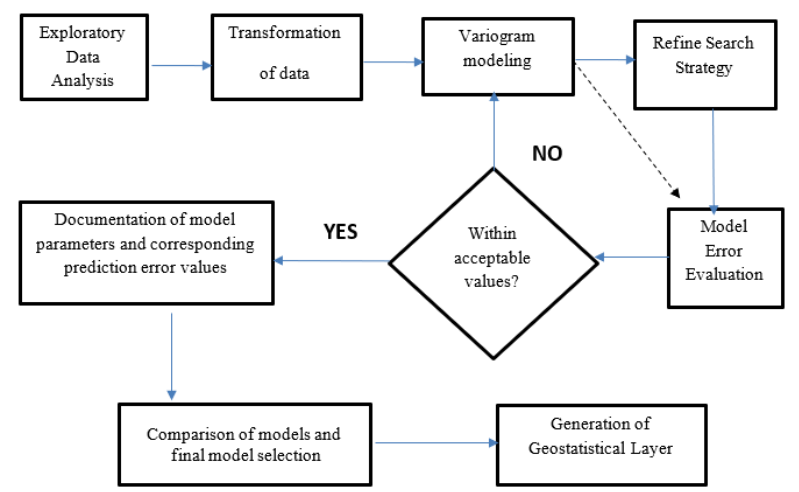

Figure 3. Geostatistical modeling workflow

\subsubsection{Exploratory Data Analysis}

Various plots such as histograms and normal quantile-quantile plots are generated to inspect and interpret the variations of $\mathrm{PM}_{10}$ concentrations generated by the dispersion model. Histograms illustrate the frequency distribution of the $\mathrm{PM}_{10}$ concentrations based on the defined number of classes and calculate summary statistics such as mean, median, maximum, and skewness. Normal QQ plots indicate univariate normality of the $\mathrm{PM}_{10}$ concentrations. If the errors of the predictions from their true values are normally distributed, the points should lie roughly along with the reference or normal line (Esri, 2003). In addition to inspecting these plots for visualizing the distribution of the $\mathrm{PM}_{10}$ concentrations, different data transformation methods are tested and assessed based on the prediction errors.

\subsubsection{Variogram Modeling}

Variograms are the primary geostatistical tool for analyzing the spatial continuity of a given attribute and can provide visualization and quantification of the dispersion and patterns of continuity or anisotropic behaviour (Paper et al., 2000). The variogram reflects some of our understanding of the geometry and continuity of the variable and can have a very important impact on predictions from numerical models (Gringarten, Deutsch, 2001). The variogram is expressed mathematically as the expected squared difference between two data values separated by a distance vector called lag or h (Tominaga, Stathopoulos, 2016). The variogram is represented by Equation 1:

$$
\gamma(h)=\frac{1}{2} E\left[(Z(x+h)-Z(x))^{2}\right.
$$

where $\gamma$ is the variogram, $\mathrm{h}$ is the lag distance and $\mathrm{Z}(\mathrm{x})$ is the data increment (Nas, 2009).

The point locations of $\mathrm{PM}_{10}$ concentrations are fitted in a theoretical semivariogram. The semivariogram is a plot of 
points, which is due to spatial autocorrelation, tend to increase in semivariance (y-axis) with increasing distance or lag (Webster, Oliver, 2001). The theoretical semivariogram is a means to explore the spatial relationship in the dataset. Based on the concept of spatial autocorrelation and the first law of geography which states that nearby things are more closely related than farther things, pairs that are close in distance should have a smaller difference than those farther away from one another. The extent to which this assumption is true can be examined in the empirical semivariogram. The variogram and modeling estimates provide a basis for interpreting the causes of spatial variation. The shape of the points in the experimental variogram can reveal much at this stage about the way that properties change with distance and the adequacy of sampling (Webster, Oliver, 2001). In this study, distance and directions are assumed to affect the variations in the data. With this assumption, the variability of the $\mathrm{PM}_{10}$ concentrations is influenced by wind directions. This is accounted for by defining the model as anisotropic. The influence of both spatial distance and directions on the dataset is investigated by enabling the Anisotropy setting in the Geostatistical Analyst tool. The values of sill, nugget and range in the semivariogram models are checked whether they change along all search directions. Directional semivariogram models are obtained to show the variations of $\mathrm{PM}_{10}$ concentrations in different distances and directions. Optimum parameters for the sill, range, and angle of direction are accounted in the results to describe the directional influence in the datasets.

In this study, all variogram model types are tested initially and it was observed that five (5) model types are closely related to the datasets and showed small prediction errors. These variogram model types are Circular, Gaussian, Exponential, Spherical and Stable. Lag size of the model is set to 15 meters, which corresponds to the grid interval of the dispersion model results. Several numbers of lags were tested with a range of values from 5 to 20 meters. The buffer from the road centerlines is set to 300 meters, thus, is set to be the maximum for the lag distance. The lag size refers to the distance of the points at which measurements of variances are taken into consideration for the semivariogram modeling. The recommended value for the lag size is usually set to a value equivalent to the interval of points in the dataset for geostatistical mapping (Hengl, 2009).

\subsubsection{Spatial Interpolation}

The interpolation process is implemented using the Kriging method, which is regarded as an optimal spatial interpolation method is a type of weighted moving average function defined by Equation 2:

$$
Z\left(X_{o}\right)=\sum_{i=1}^{n} \lambda_{i} Z\left(X_{i}\right)
$$

where $\mathrm{Z}\left(\mathrm{X}_{\mathrm{i}}\right)$ is the measured value at location $i, \lambda_{\mathrm{i}}$ is an unknown weight for the measured value at the location $I, n$ is the number of measured values and $X_{o}$ is the prediction location (Nas, 2009). The two basic implementations of Kriging interpolation are Simple Kriging (SK) and Ordinary Kriging $(\mathrm{OK})$. They primarily differ in the assumption of stationarity, which accepts that the mean, variance, and autocorrelation structure remain the same across the map extent. The SK method fully relies on this assumption, while OK assumes a constant unknown mean only over a search neighbourhood (Miller, 2017).
The spatial interpolation involves search neighbourhood parameterization, assessment of prediction errors and comparison of geostatistical models. Assigning search neighbourhood parameters in the dataset is primarily done to limit the number of points used to interpolate the values at unsampled locations. This step is based on the assumption that at a certain distance the points will no longer have a correlation with the prediction point location and it is possible that they may even be located in a different area (Esri, 2003). The advantage of assigning these search neighbourhood parameters is for computational speed, i.e. the smaller the search neighborhood, the faster the predicted values can be generated. The search mode is represented by a circle or an ellipse that can be divided into sectors (refer to Figure 4) from which an equal number of points are selected to avoid bias in a particular direction (Gringarten, Deutsch, 2001).
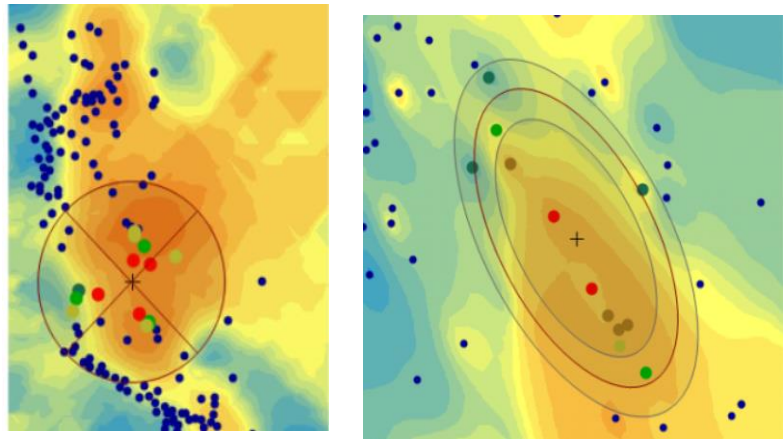

Figure 4. standard (left) and smoothing (right) search ellipses

\subsubsection{Assessment of Prediction Errors}

Prediction errors from the geostatistical models are evaluated to determine the most appropriate geostatistical model in the hourly dispersion modeling results. The optimal predictors are found to conform to these criteria: (1) mean error is close to 0 , (2) smallest value for RMSE, average standard error and mean standardized error and (3) RMS standardized error close to 1 (Nas, 2009). The RMSE, which indicates how closely the model predicts the measured values, and the mean error (ME), which is the average difference between the measured and predicted values, are determined by Equations 3 and 4:

$$
\begin{aligned}
& R M S E=\left[\frac{1}{N} \sum_{n=1}^{N}(Y-Y o)^{2}\right]^{0.5} \\
& M E=\frac{1}{N} \sum_{n=1}^{N}(Y-Y o)
\end{aligned}
$$

where $\mathrm{N}$ is the number of predicted values $\mathrm{Y}_{\mathrm{o}}$ (Enkhtur, 2013). The average standard error is equivalent to the average of the prediction standard errors while the mean standardized error pertains to the average of the standardized errors (Esri, 2003). The interpolated surface generated by the optimal predictor is also assessed if it is a good estimate or otherwise by comparing the RMSE and average standard errors. It is a good estimate if these errors are equal, an overestimate if RMSE is less than the average standard error and underestimate if RMSE is greater than the average standard error. These errors are shown with the cross-validation results. 


\section{RESULTS AND DISCUSSION}

\subsection{Exploratory Data Analysis}

Histograms and Normal QQ plots of hourly simulation results from the dispersion modeling show the spatial distribution of the pollutant concentrations. Sample histograms linked with the spatial data are illustrated in Figure 5. The histograms show that $\mathrm{PM}_{10}$ concentrations are not evenly distributed within the extent of the model. The histograms for all the simulations show that values are skewed to the left, which indicates that most of the measurements are within or below the mean concentration.

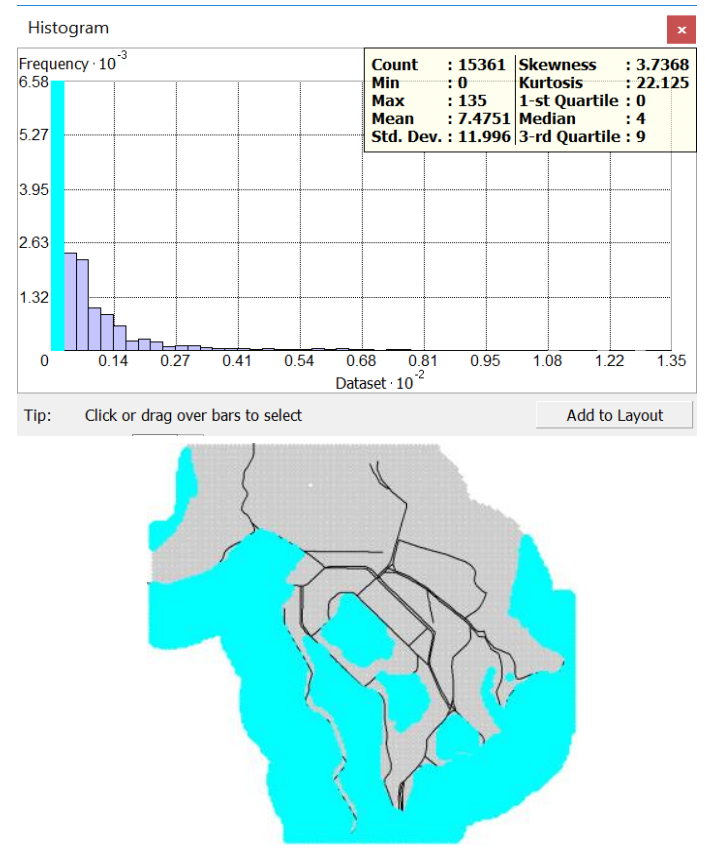

(a)

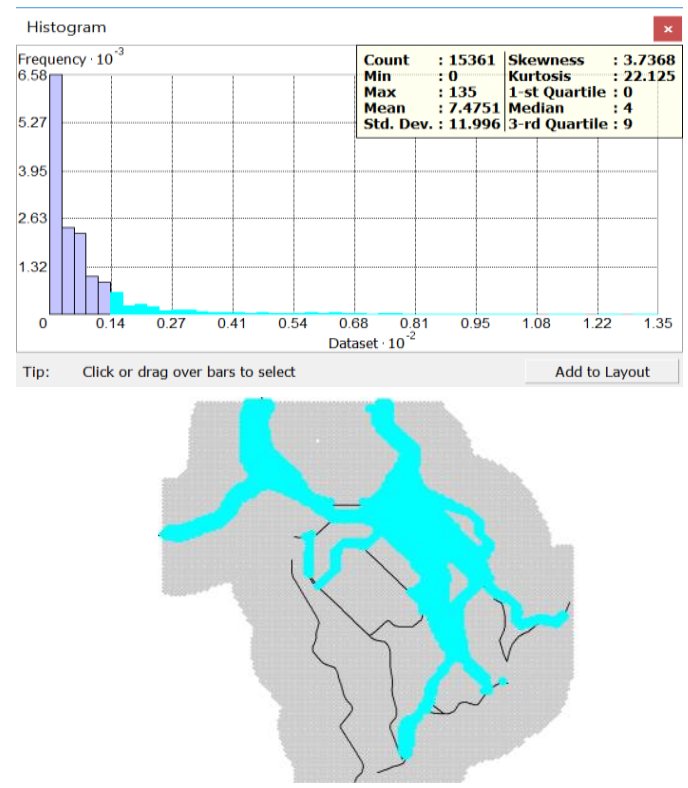

(b)

Figure 5. Histograms linked with the spatial data showing least (a) and high (b) $\mathrm{PM}_{10}$ concentrations
The Normal QQPlots, a sample of which is given in Figure 6 for daytime hour 6AM-7AM, show that the pollutant concentrations do not follow a normal curve and are consistent with the information presented by the histograms. The trend of the line shown in the Normal QQ plot is similar to exponential curves. This observation is validated by checking appropriate variogram models for the datasets.

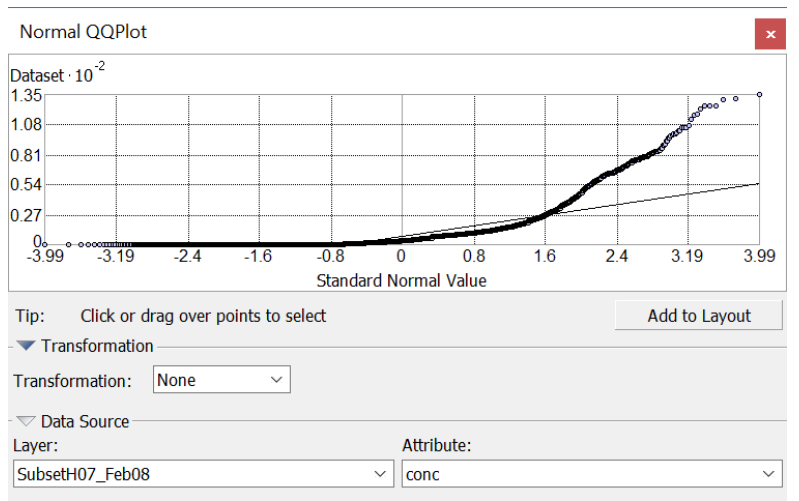

Figure 6. Normal QQPlot for daytime hour (6AM-7AM)

\subsection{Variogram Models}

Preliminary tests involve using different variogram model types with varying lag sizes (ranging from 5 to 20) and a varying number of lags (10 to 50). Results from these tests show that at a constant lag size equal to 15 and at a constant number of lags equal to 20 , prediction errors are at a minimum. Prediction errors are assessed in each test and sample results using one dataset (night-time hour 12MN-1AM) are provided in Table 1 . Highlighted in red are the values conforming to the criteria in assessing the prediction errors as discussed in section 2.2.4.

\begin{tabular}{|l|c|c|c|c|c|}
\hline Model \# & $\mathbf{1}$ & $\mathbf{2}$ & $\mathbf{3}$ & $\mathbf{4}$ & $\mathbf{5}$ \\
\hline Variogram & Circular & Spherical & Exponential & Gaussian & Stable \\
\hline Mean & 0.00032 & 0.00024 & -0.00028 & -0.00039 & -0.00038 \\
\hline RMSE & 6.84535 & 6.52962 & 5.31532 & 8.11014 & 6.92237 \\
\hline Mean Std & 0.00001 & 0.000003 & -0.00003 & -0.00005 & -0.00007 \\
\hline RMS Stdz & 0.67410 & 0.69031 & 0.75948 & 0.73452 & 0.73013 \\
\hline Ave SE & 10.16158 & 9.46694 & 7.01837 & 11.04454 & 9.48797 \\
\hline
\end{tabular}

Table 1. Preliminary test results in selecting a variogram model

The results show that the exponential variogram model produces the least errors for RMSE and average standardized error, and the highest value (closest to 1) for RMS standardized error. The model conforming to these recommended values are checked in all preliminary tests and results indicate that the most appropriate model for fitting the variograms is the exponential model.

\subsection{Spatial Interpolation}

Hourly dispersion modeling results of $\mathrm{PM}_{10}$ point concentrations spaced at 20 -meter interval show that high values range from 135 to $422 \mu \mathrm{g} / \mathrm{m}^{3}$. The dispersion modeling results show general patterns of spatial variations of the pollutant concentrations for daytime (6AM to 6PM) and nighttime (6PM to 6AM) hours. The pollutant concentrations are evident within 40 meters from the roads and are shown in a sample dataset in Figure 6. 


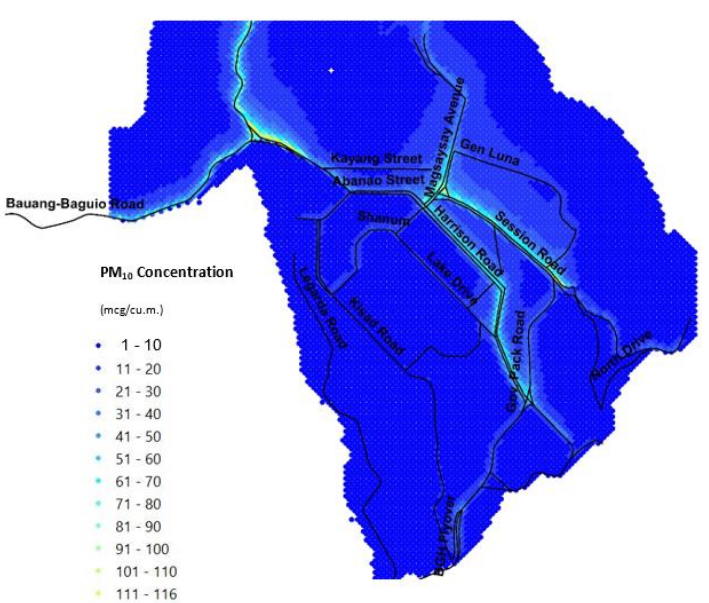

(a)

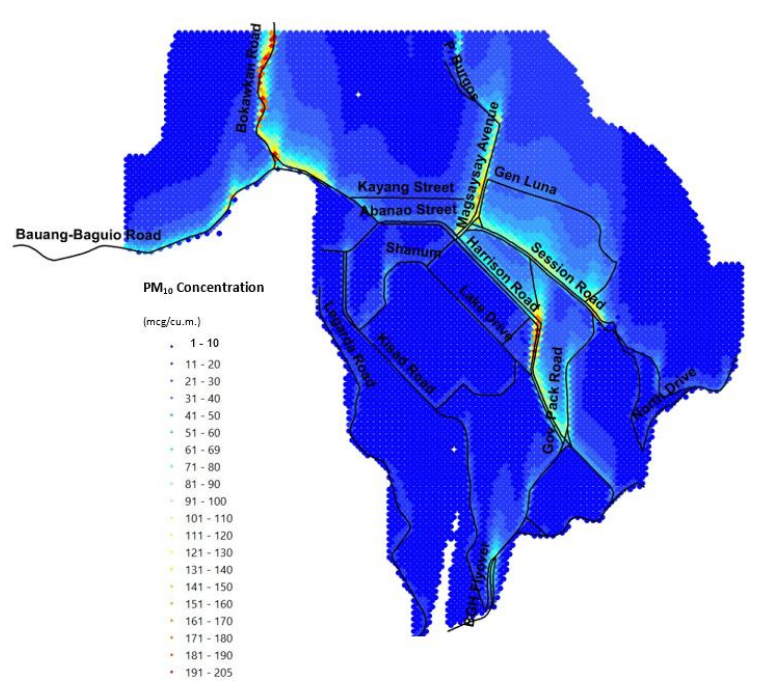

(b)

Figure 6. Dispersion modeling results for (a) daytime hour 6AM-7AM and (b) nighttime hour 6PM-7PM

Geostatistical modeling is incorporated in this study to generate finer estimates of $\mathrm{PM}_{10}$ concentrations at $5 \times 5$ meter interval. The geostatistical layers were converted to surfaces to provide continuous data on the pollutant concentration unlike the results of the dispersion model that only provide concentrations at discrete points specified in the dispersion modeling simulations. Optimal predictors for pollutant concentrations at 5-meter interval include 2 to 5 search neighbors and variable smoothing factor for night-time datasets while 2 to 10 search neighbors and smoothing factors 0.3 to 0.5 for daytime datasets. From the geostatistical modeling results (refer to Figure 7), spatial variability of $\mathrm{PM}_{10}$ concentrations is more evident on some portions of the CBD such as the surrounding areas in the north and northeast side containing road segments Bokawkan Road, Magsaysay Ave., Session Road, Gen. Luna Avenue and Harrison Road. Figure 7 shows the equivalent geostatistical layers of the dispersion model layers illustrated in Figure 6. The geostatistical layers show that trends in hourly simulations are highly dependent on the emission sources and prevailing wind conditions. Low wind conditions occur in the area, thus, there is a build-up of $\mathrm{PM}_{10}$ near the roads and disperse according to the hourly average wind conditions.

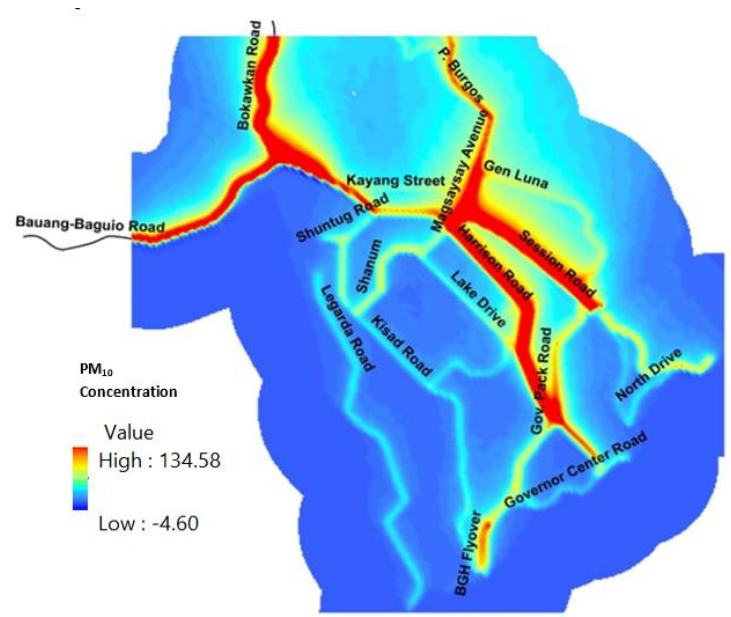

(a)

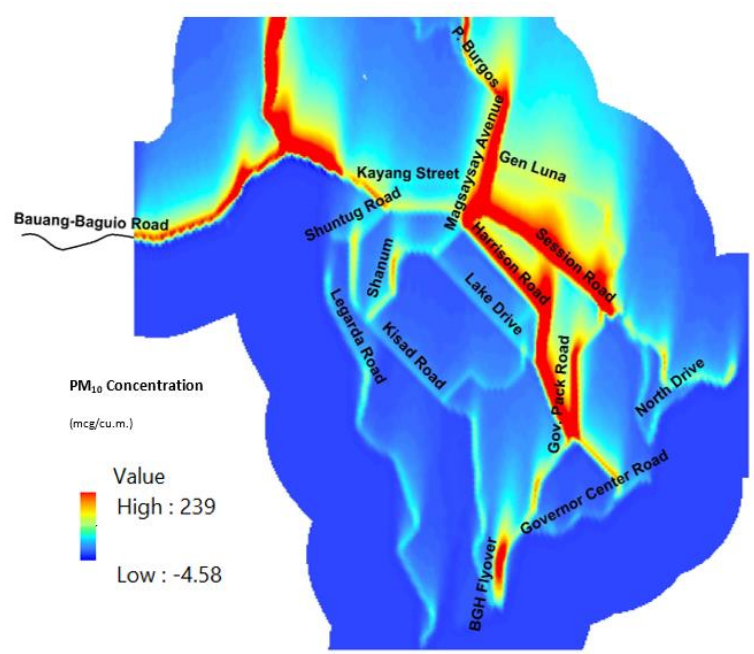

(b)

Figure 7. Geostatistical modeling results for (a) daytime hour 6AM-7AM and (b) nighttime hour 6PM-7PM

\subsection{Assessment of Prediction Errors}

Geostatistical modeling estimates are evaluated based on recommended values for mean prediction error (MPE), root mean square error (RMSE) and standardized errors. Results from several interpolation tests indicate small MPE ranging from 0.0003 to $0.0008 \mu \mathrm{g} / \mathrm{m}^{3}$ and average standardized errors 4.24 to $8.67 \mu \mathrm{g} / \mathrm{m}^{3}$. RMSE ranges from 2.95 to $5.43 \mu \mathrm{g} / \mathrm{m}^{3}$, which are approximately 2 to $3 \%$ of the maximum pollutant concentrations in the area. These small errors indicate that geostatistical methods can improve estimates of the pollutant concentrations resulting from the dispersion modeling process. Atmospheric dispersion models with modeling periods shorter than 24 hours usually yield inaccurate results with expected uncertainty of $40 \%$ when compared with in-situ data (Almazan et al., 2017)

\section{CONCLUSION}

The methodology presented in this study may be integrated with atmospheric dispersion models in refining estimates of pollutant 
concentrations, in generating surface representations and in understanding the spatial variations of the outputs from the air dispersion model simulations. The spatial and temporal variations of airborne $\mathrm{PM}_{10}$ concentrations in the $\mathrm{CBD}$ - Baguio City are assessed in this study by integrating geostatistics with dispersion modeling results. From the dispersion results, general trends can be observed on the spatial distribution of $\mathrm{PM}_{10}$ concentrations. High concentrations are evident on roads with high vehicular emissions. This observation can be seen in the hourly simulation results. However, the description of the variations is generalized because the pollutant concentrations are given at $15 \times 15$ meter spatial resolution. Pollutant concentrations may vary along the roads with widths smaller than 15 meters. Hence, geostatistical modeling is incorporated in this study to generate finer estimates of $\mathrm{PM}_{10}$ concentrations at $5 \times 5$ meter interval. Spatial variations are observed on roads with high vehicular emissions and in their surrounding areas, which are mostly located on the north and northeastern part of the study area. Temporal variations indicate that, in general, higher maximum concentrations and direction of dispersion is more evident during nighttime hours from $6 \mathrm{PM}$ to $6 \mathrm{AM}$.

\section{ACKNOWLEDGEMENTS}

The authors would like to acknowledge Dr. Mylene Cayetano and Dr. Ronald Macatangay for the valuable inputs and sharing of datasets utilized in the dispersion modeling process.

\section{REFERENCES}

Almazan, I.E.D., Racho, A., Ramos, R.V., Cayetano, M.G., 2017. Estimation of Airborne Particulate Matter from Vehicular Traffic in Quezon City using a Dispersion Model and GIS. International Conference on Air Quality and Environmental Sustainability (ICAQES) Conference Proceedings. Universiti Malaysia, Terengganu, Malaysia.

Comanda, Z., 2018. EMB cites improved air quality in Baguio City. Manila Bulletin.

Enkhtur, B., 2013. Geostatistical Modelling and Mapping of Air Pollution. ITC acedemic, p. 44.

Environmental Management Bureau (EMB), 2004. Mobilizing for Clean Air: Milestones in implementing the Clean Air Act.

Esri, 2003. ArcGIS 9 - Using ArcGIS Geosstatistical Analyst. Analysis, p. 300.

Gringarten, E., Deutsch, L., 2001. Teacher's Aide Variogram Interpretation and Modeling. Math. Geol., vol. 33, no. 4.

Guieb, M., 2015. New transport system in Baguio needed for breath of fresh air. Business Mirror.

Gulliver, J., Briggs, D., 2011. STEMS-Air: A simple GIS-based air pollution dispersion model for city-wide exposure assessment. Sci. Total Environ., vol. 409, no. 12, pp. 2419 2429.

Hagad, H. R., 2018. Source Identification and Elemental Quantification of Airborne Particulate Matter (APM) in Central Business District, Baguio City, Philippines. University of the Philippines.
Halimi, M., Farajzadeh, M., Zarei, Z., 2016. Modeling spatial distribution of Tehran air pollutants using geostatistical methods incorporate uncertainty maps. Vol. 2, no. 4, pp. 375-386.

Hengl, T., 2009. A Practical Guide to the Geostatistical Mapping, vol. 13, no. 6.

Holmes, N. S., Morawska, L., 2006. A review of dispersion modelling and its application to the dispersion of particles: An overview of different dispersion models available. Atmos. Environ., vol. 40, no. 30, pp. 5902-5928.

Locsin, J., 2014. Baguio air is among the dirtiest in the country. GMA News Online.

Miller, B. A., 2017. Geographic Information Systems and Spatial Statistics Applied for Soil Mapping: A Contribution to Land Use Management. Elsevier Inc.

Moral García, Francisco Jesús Valiente González, P López Rodríguez, F., 2010. Geostatistical Analysis and Mapping of Ground- level Ozone in a Medium Sized Urban Area. Int. J. Civ. Environ. Eng., vol. 2, no. 1, pp. 71-82.

Nas, B., 2009. Geostatistical Approach to Assessment of Spatial Distribution of Groundwater Quality. Polish J. Environ. Stud., vol. 18 , no. 6, pp. 1073-1082.

Paper, C., Model, G., Built, V.D., Morphological, A., 2000. Geostatistical models for air pollution.

Peña, R., 2018. The latest WHO pollution report. SunStar.

Pospisil, J., Jicha, M., 2010. Particulate matter dispersion modelling along urban traffic paths. Int. J. Environ. Pollut., vol. 40 , no. $1 / 2 / 3$, p. 26 .

Sertel, E., Demirel, H., Kaya, S., Demir, I., 2006. Spatial Prediction of Transport Related Urban Air Quality. Archives.

Shahraiyni, H. T., Sodoudi, S., 2016. Statistical modeling approaches for pm10 prediction in urban areas; A review of 21st-century studies. Atmosphere (Basel), vol. 7, no. 2.

Tominaga, Y., Stathopoulos, T., 2016. "Ten questions concerning modeling of near-field pollutant dispersion in the built environment. Build. Environ., vol. 105, pp. 390-402.

Webster, R., Oliver, M.A., 2001. Geostatistics for Environmental Scientists, 2nd Edition (Statistics in Practice).

Wu, E. M. Y., Kuo, S.L., 2013. A study on the use of a statistical analysis model to monitor air pollution status in an air quality total quantity control district. Atmosphere (Basel)., vol. 4, no. 4, pp. 349-364.

Xia, Q., Niu, J., Liu,X., 2014. Dispersion of air pollutants around buildings: A review of past studies and their methodologies. Indoor Built Environ., vol. 23, no. 2, pp. 201224, 2014. 